\title{
Ultrasonographic changes of submandibular glands in irradiated patients for head and neck cancers
}

\author{
C Andaloro ${ }^{*}$ Fr Gigante 2 , I Mantia 1

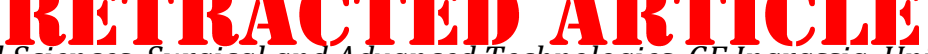 \\ ${ }^{1}$ Department of Medical Sciences, Surgical and Advanced Technologies, GF Ingrassia, University of Catania, ENT \\ Unit, Policlinico "G. Rodolico" University Hospital, Catania, Italy \\ ${ }^{2}$ Radiotherapy Unit, Belcolle Hospital, Viterbo, Italy
}

\section{- Original article}

\author{
*Corresponding authors: \\ Claudio Andaloro, M.D., \\ E-mail: \\ cla.anda@gmail.com
}

Revised: March 2020

Accepted: April 2020

Int. J. Radiat. Res., January 2021; 19(1): 127-134

DOI: 10.29252/ijrr.19.1.127

\begin{abstract}
Background: Salivary glands (SGs) are usually irradiated during radiotherapy for head and neck cancers, which may lead to radiation-induced changes. These changes could be evaluated safely by ultrasonography; however, there have been few studies in this regard. Thus, we aimed to investigate the changes in post-radiotherapy submandibular glands using ultrasonography in patients undergoing head-neck radiotherapy. Materials and Methods: We evaluated 46 submandibular glands of 23 patients ultrasonographically in terms of echogenicity, echotexture, margin, and dimensional (anteroposterior, superoinferior, mediolateral length and volume) changes before radiotherapy, and at the second and sixth months after radiotherapy. Results: About $93.5 \%$ of the submandibular glands were hyperechoic before RT. When the same submandibular glands were evaluated two months later, $39.1 \%$ were hyperechoic. Six months after the therapy, 56,5\% were hyperechoic $(p<0.001)$. There is also a similar trend for the echotexture $(p<0.001)$ and the regularity margins before RT, two months after-, and at sixth month after RT $(p<0.001)$. Also, there were statistically significant differences between anteroposterior, superoinferior, mediolateral lengths, and volumetric values measured before radiotherapy and at the end of two and six months after radiotherapy onset $(p<0.001)$. Conclusion: We revealed that submandibular glands were isoechoic or hypoechoic, heterogeneous, and irregular after radiotherapy; however, as the time elapsed after radiotherapy, the parenchyma structure returned to normal in half of them. Indeed, defining radiotherapy-related changes in SGs may be helpful in better understanding the mechanism of common oral complications associated with $\mathrm{RT}$, and in the differential diagnosis of other SG diseases.
\end{abstract}

Keywords: Salivary gland, Radiotherapy, Head and neck cancer, Ultrasound.

\section{INTRODUCTION}

Radiotherapy (RT) is an integral tool in the management of head and neck cancers (1). Nevertheless, despite the advancement in technology and clinical knowledge, the use of RT for head and neck malignancies can cause major salivary gland (SG) damages because SG parenchyma is very sensitive to radiation, particularly the parotid gland (2). Changes in the SGs depend on the volume and radiation dosage of the gland in the radiation field, but the mechanisms of these changes are unclear $(2,3)$.

SG damage after RT includes lymphocytic infiltration, degeneration, necrosis, acinar cell loss, atrophy, fibrosis, and duct dilatation (4). Moreover, reductions in the permeability and blood flow may be present (5). There have been reports in the literature to accurately assess the effects of head and neck RT on SGs. For this purpose, there are various techniques such as survey, histological evaluation, sialometry, 
computed tomography, positron emission tomography, magnetic resonance imaging, sialography, scintigraphy, and ultrasonography (USG) (3).

USG is the preferred method worldwide for imaging soft tissues, especially the SGs (6). USG provides detailed information about the morphological characteristics of the SGs (parenchyma structure, size, and volume) and their relationship with the surrounding anatomical structures (7). USG allows the examination of the entire submandibular and sublingual glands and the superficial lobe of the parotid gland (8).

RT can change the normal morphology (echotexture, echogenicity, and margin) and size of the SGs along the radiation pathway. USG can monitor all these changes: homogenous to heterogeneous, hyperechoic to hypoechoic, regular to irregular, and diminished in size (2); however, there have been few studies in this regard, especially concerning ultrasound evaluation of post-RT changes in the submandibular glands.

This study aimed to investigate bilateral submandibular gland changes (echogenicity, echotexture, margin, size, and volume) in patients with head and neck cancers who underwent RT using USG.

\section{MATERIALS AND METHODS}

\section{Ethical statement}

We have conducted this study in the Department of Radiology and Radiotherapy of Belcolle Hospital, Viterbo, Italy with the approval of the Local Ethics Committee dated 21.02.2019 and numbered 65EK1. Additionally, the researchers notified the participants about the study and obtained written and oral informed consents. All methods were conducted in accordance with the relevant approved regulations, guidelines, and declaration of Helsinki.

\section{Work Plan}

Since the submandibular glands could be more easily visualized than the parotid glands, this study aimed to investigate the changes in submandibular glands by USG before and after RT in patients undergoing head and neck RT at the Department of Radiology and Radiotherapy of Belcolle Hospital, Viterbo, Italy.

In recent years, our hospital has used intensity-modulated radiotherapy (IMRT) for patients with head and neck tumors to enable preservation of the major salivary glands. Thus, we ultrasonographically evaluated the submandibular glands of patients in terms of echogenicity, echotexture, margin, and dimensional (anteroposterior, superoinferior, mediolateral length, and volume) changes before RT and at the second and sixth months after RT onset. Examination of the submandibular gland with the help of USG is more straightforward because of its anatomical position and size ${ }^{(9)}$.

\section{Patients' Selection}

We have included 23 patients with histologically proven head and neck cancer and treated with RT. However, we excluded patients with a history of background SG disease, including SG malignancy or Sjögren's syndrome, Mikulicz syndrome, sarcoidosis, history of HIV or autoimmune diseases, or history of the previous RT or surgical procedures in the head and neck region.

\section{RT Application}

All patients, who planned to undergo RT, underwent computed tomography (CT) scan for planning by providing appropriate immobilization with a thermoplastic mask. We combined the three-dimensional conformal planning system, CT images, positron emission tomography (PET)-CT, and magnetic resonance imaging (MRI) images of the patients to measure the target volumes, such as gross tumor volume, clinical target volume, and planned target volume. We determined the most suitable RT areas for each patient using the Eclipse Treatment Planning System (Version 11, Varian Medical Systems, Palo Alto, CA). Clinicians performed RT using the standard procedure: 1.8 -3 Gy daily for 5 days/week and a total of 30-70 
Gy using the linear accelerator.

\section{Demographic characteristics}

We recorded personal information (name, surname, identification number, age, place of birth, and telephone) of the patients and collected a detailed medical and dental history through records of past therapies, general systemic conditions, medications used, and RT treatment plans.

\section{USG procedure}

We imaged the submandibular glands of all the patients extraoral and bilaterally (46 submandibular glands total) using the Toshiba Aplio 300 USG device (Toshiba Medical Systems Corporation, Tokyo, Japan) by placing the probe parallel to the lower edge of the mandible and vertically to the body of the mandible in two planes perpendicular to each other. All procedures were performed and interpreted by one experienced senior ultrasound physician, who was not involved in this study, at the same institution for all patients.

\section{SG assessment}

We evaluated the parenchyma of the submandibular glands as hyperechoic, isoechoic, or hypoechoic in terms of echogenicity compared to the echogenicity of the surrounding soft tissue, such as masseter muscle (3). The parenchyma structure showing brighter echogenicity than the surrounding soft tissues was hyperechoic, the parenchyma structure showing the same echogenicity as the surrounding soft tissue was isoechoic, and the parenchymal structure showing darker echogenicity than the surrounding soft tissues was hypoechoic echogenicity. We considered the parenchyma of the submandibular glands as homogeneous or heterogeneous in terms of echotexture. The evaluations classified the margins of the submandibular glands as regular or irregular.

As a dimensional measurement of submandibular glands, we performed anteroposterior, superoinferior, mediolateral length measurements, and volume measurements as following: on the USG image obtained with the probe positioned parallel to the lower edge of the mandible, we defined the largest dimension of the submandibular gland measured in the anteroposterior direction as "anteroposterior length"; on the USG image obtained with the probe positioned parallel to the lower edge of the mandible, we defined the largest dimension of the submandibular gland measured in the superoinferior direction as "superoinferior length"; on the USG image obtained with the probe positioned vertically to the body of the mandible, the study defined the largest dimension of the submandibular gland measured in the mediolateral direction as "mediolateral length"; we performed volume calculations after the measurements of anteroposterior length, superoinferior length, and mediolateral length on the USG image of the submandibular gland (figure 1). We calculated the tab automatically. Evaluation of the parenchyma in terms of eco-texture, anteroposterior and superoinferior length measurements of submandibular glands used in this study are shown in figure 1.

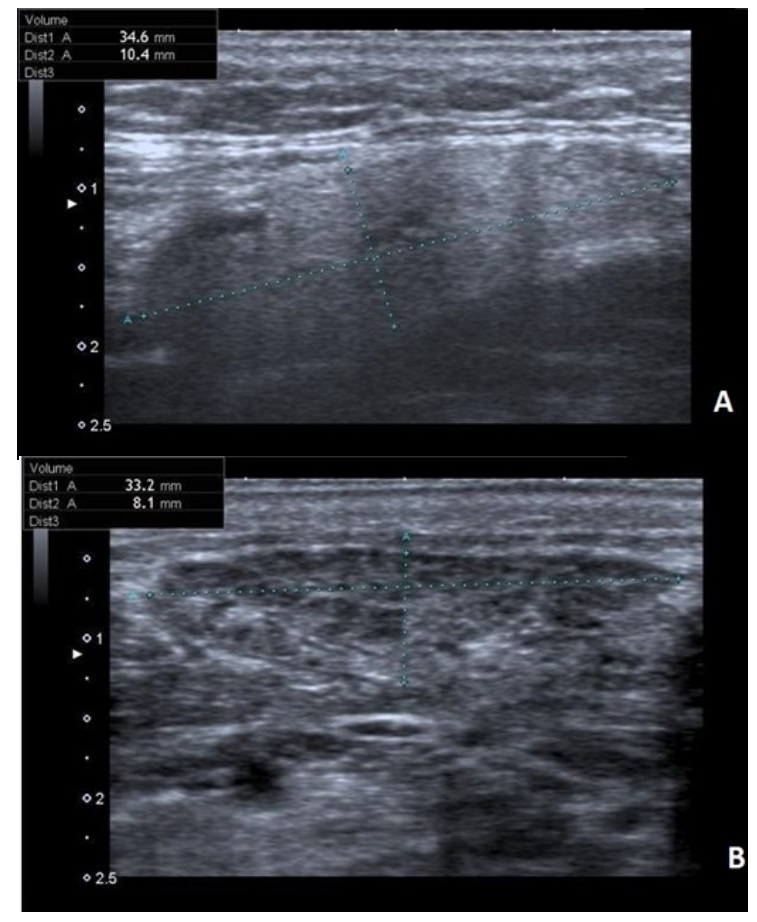

Figure 1. Evaluation of eco-texture, anteroposterior and superoinferior length of submandibular gland. A.

Submandibular gland pre-RT with homogeneous ecotex and normal length. B. Submandibular gland post-RT with heterogeneous ecotex and abnormal length. 


\section{Statistical analysis}

The study used SPSS Version 20 (IBM, SPSS Inc., Chicago, IL) statistical study program for statistical analysis of the collected data. The skewness and kurtosis tests were used for testing the normal distribution of continuous variables. Frequencies and percentages were used to summarize categorical variables. We used the Chi-square test to compare the echogenicity, echotexture, and margin of the submandibular glands before RT, in the second month of RT, and at the sixth month of RT. We then used a repeated measurements ANOVA test to compare dimensional measurements of submandibular glands before RT, the second month after RT, and the sixth month after RT. When the p-value was $<0.05$, the study used a Bonferroni Post-Hoc test to determine the difference between the groups. Results were considered significant with $\mathrm{p}<0.05$.

\section{RESULTS}

Our study included 46 bilateral submandibular glands of 23 patients, 21 $(91.3 \%)$ of which were male and $2(8.7 \%)$ of which were female. The ages of our patients ranged from 28 to 83 years, with a mean age of $53.5 \pm 12.6$ years. In the treatment plans applied to the patients, the mean number of fractions was $28 \pm 6.6$ with a range of 13 to 36 . The daily radiation dose ranged from 1.8 to 3 Gy (mean $2.04 \pm 0.22 \mathrm{~Gy}$ ), and the total radiation dose ranged from 30 to 75 Gy (mean 60.98 \pm 13.61 Gy), (table 1). When we evaluated the echogenicity of the submandibular glands before RT, $43(93.5 \%)$ of 46 submandibular glands were hyperechoic, 1 (2.2\%) isoechoic, and 2 $(4.3 \%)$ were hypoechoic. When the same submandibular glands were evaluated two months later, 18 (39.1\%) were hyperechoic, 21 (45.7\%) were isoechoic, and 7 (15.2\%) were hypoechoic. Six months after the therapy, 26 were hyperechoic, 16 (34.8\%) were isoechoic, and $4(8.7 \%)$ were hypoechoic $(\mathrm{p}<0.001)$.
Moreover, the echotexture of 46 submandibular glands showed that 42 (91.3\%) were homogenous and 4 (8.7\%) were heterogeneous before the RT. At the second month after RT, 16 (34.8\%) were homogenous and $30(65.2 \%)$ were heterogeneous. In the sixth month after RT, 30 (65.2\%) were homogenous and $16(34.8 \%)$ were heterogeneous $(\mathrm{p}<0.001)$, (table 2).

When we examined the margins of the submandibular glands, 41 (89.1\%) were regular and $5(10.9 \%)$ were irregular before RT. Two months after RT, 7 (15.2\%) were regular and 39 (84.8\%) were irregular. At six months, 24 $(52.2 \%)$ were regular and $22(47.8 \%)$ were irregular $(\mathrm{p}<0.001)$, (table 2).

The mean anteroposterior length of 46 submandibular glands was $32.39 \pm 4.55 \mathrm{~mm}$ before radiotherapy, $30.38 \pm 4.8 \mathrm{~mm}$ in the second month after RT, and $31.5 \pm 3.68 \mathrm{~mm}$ in the sixth month. Additionally, the statistical analysis found significant differences between anteroposterior lengths measured at different time intervals $(\mathrm{p}<0.01)$. The mean superoinferior length of 46 submandibular glands included in the study was $9.96 \pm 1.54 \mathrm{~mm}$ before RT, $8.76 \pm 1.26 \mathrm{~mm}$ in the second month after RT, and $9.08 \pm 1.01 \mathrm{~mm}$ in the sixth month after RT $(\mathrm{p}<0.001)$, (table 3$)$. The post-hoc test results found that there was a statistically significant difference between the measurements taken before the RT and the second and sixth months after the initiation of RT. However, there was no statistically significant difference between the measurements performed in the second and sixth months after the initiation of RT.

The mean mediolateral length of 46 submandibular glands included in the study was $24.66 \pm 3.77 \mathrm{~mm}$ before RT, $22.03 \pm 3.73 \mathrm{~mm}$ in the second month after RT, and $21.76 \pm 4.01 \mathrm{~mm}$ in the sixth month after RT.

The mean volume of 46 submandibular glands included in the study was $4.21 \pm 1.01 \mathrm{~cm}^{3}$ before RT, $3.08 \pm 0.77 \mathrm{~cm}^{3}$ in the second month after RT, and $3.32 \pm 0.63 \mathrm{~cm}^{3}$ in the sixth month post-RT $(\mathrm{p}<0.001)$, (table 3$)$. 
Table 1. Demographic details of study population.

\begin{tabular}{|c|c|}
\hline Characteristics & Data \\
\hline \multicolumn{2}{|c|}{ Age, in years } \\
\hline Mean \pm SD & $53.5 \pm 12.6$ \\
\hline Median & 52 \\
\hline Range & $28-83$ \\
\hline \multicolumn{2}{|c|}{ Gender, number (\%) } \\
\hline Male & $21(91.3 \%)$ \\
\hline Female & $2(8.7 \%)$ \\
\hline \multicolumn{2}{|c|}{ Cancer types, number (\%) } \\
\hline Larynx cancer & $14(60.9 \%)$ \\
\hline Lymphoma & $4(17.4 \%)$ \\
\hline Nasopharynx cancer & $2(8.8 \%)$ \\
\hline Hypopharynx cancer & $1(4.3 \%)$ \\
\hline Maxillary sinus tumor & $1(4.3 \%)$ \\
\hline Lower lip cancer & $1(4.3 \%)$ \\
\hline \multicolumn{2}{|c|}{ Daily radiation dose, in Gy } \\
\hline Mean \pm SD & $2.04 \pm 0.22$ \\
\hline Range & $1.8-3$ \\
\hline \multicolumn{2}{|c|}{ Total radiation dose, in Gy } \\
\hline Mean \pm SD & $60.98 \pm 13.61$ \\
\hline Range & $30-75$ \\
\hline
\end{tabular}

\section{DISCUSSION}

In our study, we aimed to investigate the changes in the bilateral submandibular glands (echogenicity, echotexture, margin, size, and volume) of patients diagnosed with head and neck cancer undergoing RT using USG. The severity of RT-related complications varies depending on factors such as type of RT, total radiation dose, fraction dose, treatment area limits, and duration of treatment $(10,11)$.

In summary, we documented that the submandibular gland parenchyma had normal hyperechoic echogenicity, homogenous echotexture, and a regular margin before RT, whereas it was seen as isoechoic or hypoechoic, heterogeneous, and irregular after RT; however, as the time elapsed after RT, the parenchyma structure returned to normal in about half of the glands. These changes in echogenicity, echotexture, margin, and dimensional measurements (anteroposterior length, superoinferior length, mediolateral length, and volume) of the submandibular glands appear to be consistent with the findings of most studies in the literature $(2,5,12-13)$.

Firstly, the total radiation dose, in particular, is an important factor in SG damage due to RT $(11,14)$. In our study, the total radiation dosage of the selected patient groups was between 30 to 75 Gy. The mean total radiation dose was $60.98 \pm 13.61 \mathrm{~Gy}$. This dose is within the limits of the volumetric changes in the SGs, which is one of the main parameters of our study. Bronstein et al. (15), in their study with CT scans, found that the increased image density in the SGs is associated with a high radiation dose (45 Gy) while Nagler et al reported that parotid glands exposed to radiation of $\geq 60$ Gy could lead to permanent damage to acinar cells (16). In some 
studies where the morphological changes in SGs were proportionate to the radiation dose $(5,17)$, researchers found that there was a high correlation between the decrease in gland volume and the average total radiation dose. When radiation therapy applied a total radiation dose of 60 Gy due to head and neck cancer, SG function loss is close to $80 \%$ and is generally irreversible (18). The parenchymal structure of the SGs is very sensitive to radiation, and this change is an expected result considering the total radiation doses applied to our patients.

Secondly, according to our findings of the echogenicity, while submandibular glands had hyperechoic echogenicity in $93.5 \%$ before RT, this rate decreased to $39.1 \%$ in the second month after RT and increased to $56.5 \%$ in the sixth month after RT. These findings show that after RT, the submandibular gland echogenicity changes from hyperechoic to iso-hypoechoic, but as time goes on (six months), approximately one-third of the glands showing isoechoic and hypoechoic echogenicity change back to normal gland parenchyma. As a result of the study conducted by Cheng, although the echogenicity of submandibular glands was generally hyperechoic in healthy individuals and hypoechoic in patients treated with RT, no statistically significant difference was found between the two groups (19).

Thirdly, for our ecotexture findings, while submandibular glands had a homogenous echotexture of $91.3 \%$ before $\mathrm{RT}$, this rate decreased to $34.8 \%$ in the second month after the initiation of RT and increased to $65.2 \%$ in the sixth month after the initiation of RT. These data show that, after RT, the submandibular gland echotexture changes from homogeneous to heterogeneous; but as time increases (about six months), approximately half of the glands showing heterogeneous echotexture changes revert back to normal gland parenchyma. The SG echotexture after RT generally has a heterogeneous appearance, and hyperechoic lines/spots and hypoechoic areas representing fibrosis and inflammatory response are the causes of this heterogeneous appearance in the gland parenchyma ${ }^{(1)}$. In the study by Dost and Kaiser, they observed the echotexture of healthy
SGs homogeneously (20), while in the study conducted by Jindal et al., the SGs echotexture varied from homogenous to heterogeneous after RT (21). Similarly, in a study by Yang et al, of the homogeneous parenchymal structure of 14 healthy parotid glands, the study found 24 parotid glands treated with RT to be significantly heterogeneous (1). Imanimognaddam et al. showed that the parenchyma of both parotid and submandibular glands was homogeneous in the first stage of USG, but this difference was different between echotextures in the later stages, and this difference was statistically significant (2). Furthermore, other studies reported similar findings (8). In a study by Cheng, submandibular glands of healthy individuals had $90 \%$ homogeneous echotexture (19), while the study observed $28 \%$ homogenous and $72 \%$ heterogeneous echotexture in patients treated with RT. In both SGs, there was a statistically significant difference in terms of echotexture of healthy and RT groups.

For the margin analysis, while the rate of submandibular gland with regular margins was $89.1 \%$ before RT, this rate decreased to $15.2 \%$ in the second month after RT and increased to $52.2 \%$ in the sixth month after RT. These findings indicate that submandibular gland margins have changed from regular to irregular two months after RT, but that half of the glands showing irregular margin change have returned to normal at six months after the therapy. In the study, conducted by Imanimognaddam et al, the margins of both parotid and submandibular glands showed irregular changes after RT, and the difference between gland margins observed at different USG stages was statistically significant (2). In a study performed on the submandibular glands of patients with diagnosed head and neck cancer who received RT, Cheng observed that submandibular glands generally had regular margins in the control group and irregular margins in the patient group (19).

Moreover, there were statistically significant differences between anteroposterior, superoinferior, and mediolateral lengths of the submandibular glands measured before radiotherapy and at the end of two and six 
months after radiotherapy onset.

Regarding the volume, the mean submandibular glands volume was smaller in the second month after the onset of RT compared to pre-RT measurements. Measurements made during the sixth month showed an increase compared to the second month. Although the findings of the submandibular glands measured in our study were different, the selected imaging method and SGs examined were in parallel with the findings of most of the previous studies $(5,12)$. In contrast to the study by Johari et al. (22), reductions in submandibular gland sizes were found to be statistically significant in most studies $(2,19,21)$. This is a possible result due to submandibular glands remaining in the radiation field. Reduction in gland size and volume is a result of acinar atrophy and fibrous changes in SGs exposed to radiation (21). In the study performed by Cheng, both the parotid and submandibular glands were significantly smaller in RT patients compared to healthy subjects (19). Jindal et al observed a significant reduction in the size (length, width, and depth) of the SGs after RT in 20 patients diagnosed with head and neck cancer (21). In another study by Johari et al., a reduction in the length and width of both parotid and submandibular glands was found 6 to 7 weeks after RT; however, only the decrease in parotid gland width was statistically significant (22). Imanimognaddam et al observed a substantial reduction in parotid and submandibular gland sizes after RT in 20 patients with head and neck malignancy (2).

Indeed, RT plays a vital role in improving local control and survival in patients diagnosed with head and neck cancer (1). Oral complications due to RT may cause disruption of cancer treatment, discontinuation of treatment, increase in cost, and/or decrease in patient's quality of life (23). Therefore, it is crucial to identify, treat, and prevent oral complications.

In recent years, SG dysfunction has become a growing problem in patients diagnosed with head and neck cancer undergoing RT (24). Numerous studies have inspired us to better understand the SG dysfunction due to RT and have influenced the development of new therapeutic strategies. These studies should include studies on radiation-induced changes in SGs (25).

Indeed, accurate evaluation and investigation of morphological and functional changes in the SG after RT may help clinicians better understand the mechanism of common oral complications, such as SG dysfunction and xerostomia due to RT. Also, defining RT-related changes in the SGs has an important role in the differential diagnosis of other SG diseases (3).

Of note, we preferred the use of USG in our study, as in many studies in the literature $(2,5,19$, $21,22)$, because of its high sensitivity and specificity values for both SG examination and significant advantages. USG examination, which is comfortable and accessible, and most importantly does not contain ionizing radiation, has enabled us to follow a safer route for our patients. The reason for our evaluation at three different times was to determine whether the changes in the SGs due to RT were reversible; in other words, to determine the amount of permanent damage. However, our study does not include long-term (after six months) follow-up due to time limitation. Long-term follow-up of the patients can determine the long -term changes and complications after RT. Also, the number of patients included in the study was limited due to time limitation.

\section{CONCLUSION}

The present study may provide additional insight into the effects of radiation-induced salivary gland injury, we revealed that the submandibular gland parenchyma had normal hyperechoic echogenicity, homogenous echotexture, and a regular margin before RT, whereas it was seen as isoechoic or hypoechoic, heterogeneous and irregular after RT; however, as the time elapsed after RT, the parenchyma structure returned to normal in about half of the glands. To avoid misinterpretation of ultrasound images, post-RT changes in the sonographic appearance of parotid glands should be taken into account in ultrasound examination of head and neck cancer patients with previous facial 
RT. Moreover, defining RT-related changes in SGs may be helpful in better understanding the mechanism of common oral complications associated with RT, as well as in the differential diagnosis of other SG diseases.

\section{Conflicts of interest: Declared none.}

\section{REFERENCES}

1. Yang X, Tridandapani S, Beitler JJ, Yu DS, Yoshida EJ, Curran WJ, et al. (2012) Ultrasound histogram assessment of parotid gland injury following head-and-neck radiotherapy: a feasibility study. Ultrasound Med Biol, 38(9): 1514-1521.

2. Imanimoghaddam M, Rahrooh M, Tafakhori Z, Zahedanaraki S, Homaeieshandiz $F$ (2012) Changes of parotid and submandibular glands caused by radiotherapy--an ultrasound evaluation. Dentomaxillofac Radiol, 41(5): 379-384.

3. Cheng SCH, Wu VWC, Kwong DLW, Ying MTC (2011) Assessment of post-radiotherapy salivary glands. Br J Radiol, 84 (1001): 393-402.

4. Redman RS (2017) Histologic changes in the salivary glands following radiation therapy In: Salivary gland development and regeneration, (Cha S, ed.), Berlin: Springer International Publishing, Berlin, Germany.

5. Wu VWC, Ying MTC, Kwong DLW (2011) Evaluation of radiation-induced changes to parotid glands following conventional radiotherapy in patients with nasopharygneal carcinoma. Br J Radiol, 84(1005): 843-849.

6. Gokhale S (2017) Sonography of salivary glands. Ultrasound Med Biol, 43: S177.

7. Ashwini, Shankar VN, Praveena V, Amingad BB (2014) Ultrasonography of salivary gland: A pictorial review. J Oral Maxillofac Surg Med Pathol, 26(1): 61-67.

8. Gritzmann N, Rettenbacher T, Hollerweger A, Macheiner P, Hubner E (2003) Sonography of the salivary glands. Eur Radiol, 13(5): 964-975.

9. Onkar D and Onkar P (2016) Submandibular gland biometry by high frequency ultrasound. J Anat Soc India, 65(2): 156158.

10. Palma LF, Gonnelli FAS, Marcucci M, Dias RS, Giordani AJ, Segreto RA, et al. (2017) Impact of low-level laser therapy on hyposalivation, salivary $\mathrm{pH}$, and quality of life in head and neck cancer patients post-radiotherapy. Lasers Med Sci, 32(4): 827-832.

11. Deasy JO, Moiseenko V, Marks L, Chao KC, Nam J, Eisbruch A (2010) Radiotherapy dose-volume effects on salivary gland function. Int J Radiat Oncol Biol Phys, 76: S58-S63.

12. Wang ZH, Yan C, Zhang ZY, Zhang CP, Hu HS, Kirwan J, et al. (2009) Radiation-induced volume changes in parotid and submandibular glands in patients with head and neck cancer receiving postoperative radiotherapy: A longitudinal study. Laryngoscope, 119(10): 1966-1974.

13. Tomitaka E, Murakami R, Teshima K, Nomura T, Nakaguchi $Y$, Nakayama $H$, et al. (2011) Longitudinal changes over 2 years in parotid glands of patients treated with preoperative 30-gy irradiation for oral cancer. Jpn J Clin Oncol, 41 (4): 503-507.

14. Li Y, Taylor JM, Haken RKT, Eisbruch A (2007) The impact of dose on parotid salivary recovery in head and neck cancer patients treated with radiation therapy. Int J Radiat Oncol Biol Phys, 67(3): 660-669.

15. Bronstein A, Nyberg D, Schwartz A, Shuman W, Griffin B (1987) Increased salivary gland density on contrastenhanced CT after head and neck radiation. AJR Am J Roentgenol, 149(6): 1259-1263.

16. Nagler RM, Baum BJ, Fox PC (1993) Effects of X irradiation on the function of rat salivary glands at 3 and 40 days. Radiat Res, 136(3): 392-396.

17. Teshima K, Murakami R, Tomitaka E, Nomura T, Toya R, Hiraki A, et al. (2009) Radiation-induced parotid gland changes in oral cancer patients: correlation between parotid volume and saliva production. Jpn J Clin Oncol, 40(1): 42-46.

18. Harrison JS, Dale RA, Haveman CW, Redding SW (2003) Oral complications in radiation therapy. Gen Dent, 51(6): 552-560.

19. Cheng $\mathrm{CH}$ (2011) Ultrasound evaluation of salivary and thyroid glands in patients treated with external beam radiotherapy for nasopharyngeal carcinoma [dissertation]. The Hong Kong Polytechnic University, Hong Kong.

20. Dost $P$, Kaiser $S$ (1997) Ultrasonographic biometry in salivary glands. Ultrasound Med Biol, 23(9): 1299-1303.

21. Jindal R, Ahmed J, Shenoy N, Rai S, Ongole R, Gupta A (2015) Ultrasonographic changes in salivary glands following radiotherapy. Int J Res Stud Biosci, 3(4): 112-115.

22. Johari M, Rashid RJ, Jangjoo AG, Esmaeili F, Razavi HR, Reyhani Z, et al. (2016) Sonographic assessment of parotid and submandibular glands in patients undergoing $3 \mathrm{~d}$ conformal radiotherapy. Int J Med Res Health Sci, 5(6): 127135.

23. Lalla R, Treister N, Sollecito $T$, Schmidt B, Patton L, Mohammadi K, et al. (2017) Oral complications at 6 months after radiation therapy for head and neck cancer. Oral Dis, 23(8): 1134-1143.

24. Tuomikoski L, Kapanen M, Collan J, Keyriläinen J, Saarilahti K, Loimu V, et al. (2015) Toward a more patient-specific model of post-radiotherapy saliva secretion for head and neck cancer patients. Acta Oncol, 54(9): 1310-1316.

25. Grundmann O, Mitchell G, Limesand K (2009) Sensitivity of salivary glands to radiation: from animal models to therapies. J Dent Res, 88(10): 894-903. 